\title{
Ueber die Bewegung eines Rotationskörpers in einer Flüssigkeit.
}

\author{
(Von Herrn G. Kirchhoff in Heidelberg.)
}

In ihrem Buche Treatise on natural philosophy, Oxford 1867, p. 264 haben die Herren Thomson und Tait die Aufgabe behandelt, die Bewegung eines Rotationskörpers in einer Flüssigkeit zu bestimmen. Unter den Bedingungen, unter denen sie dieselbe gelöst haben, befindet sich auch die, dass der Körper um seine Rotationsaxe nicht rotirt und diese Axe in einer festen Ebene bleibt. Ich habe gefunden, dass die Aufgabe auch ohne diese Beschränkung lösbar ist und ebenso, wie unter derselben, auf elliptische Integrale führt. Das zu zeigen ist der Zweck dieser Abhandlung.

Die Differentialgleichungen der Bewegung sollen unter den folgenden Voraussetzungen abgeleitet werden: Ein starrer Körper von beliebiger Gestalt und beliebig vertheilter Masse befindet sich in einer incompressibeln, homogenen Flüssigkeit, die ausser durch seine Oberfläche durch eine im Unendlichen liegende, geschlossene, feste Fläche begrenzt ist. Reibung findet in der Flüssigkeit nicht statt. Auf den Körper wirken Kräfte, welche ein Potential besitzen, das nur von der Lage des Körpers abhängig ist. Auf die Theile der Flüssigkeit wirken keine Kräfte. Wirbelbewegungen sind in der Flüssigkeit nicht vorhanden. Die Geschwindigkeiten ändern sich überall in derselben stetig mit den Coordinaten des Ortes; durch diese Annahme wird der Fall ausgeschlossen, dass von der Oberfläche des Körpers eine Fläche ausgeht, die - ähnlich der Oberfläche eines Strahles, der in gleichartiger Flüssigkeit sich bewegt - Theile der Flüssigkeit trennt, die verschiedene Geschwindigkeiten haben. Endlich soll der Bewegungszustand des Systems aus dem Zustande der Ruhe durch Kräfte, die auf den Körper wirkten, hervorgegangen sein; diese Annahme bildet eine Beschränkung der Aufgabe in dem Falle, dass der von der Flüssigkeit erfüllte Raum ein mehrfach zusammenhẳngender ist, dass der Körper z. B. die Gestalt eines Ringes hat.

Um die Integration der Differentialgleichungen, die unter diesen Voraüssetzungen gelten, zu ermöglichen, wird dann weiter angenommen werden, 
238 Kirchhoff, über die Bewegung eines Rotationskörpers in einer Flüssigkeit.

dass die Kräfte, die auf den Körper wirken, verschwinden, dass die Oberfläche des Körpers eine Rotationsfläche und die Vertheilung der Masse in ihm symmetrisch zur Rotationsaxe ist.

\section{\$. 1.}

Die in Rede stehenden Differentialgleichungen sollen aus dem Hamiltonschen Principe entwickelt werden. Bezeichnet $\Omega$ das Potential der auf den Körper wirkenden Kräfte, $T$ die lebendige Kraft des ganzen Systemes und $t$ die Zeit, so ist nach diesem:

$$
\text { (1.) } \delta \int(\Omega+T) d t=0 \text {. }
$$

Es seien $x, y, z$ die Coordinaten eines Punktes des Körpers in Bezug auf ein in diesem festes Coordinatensystem, $\xi, \eta, \zeta$ die Coordinaten desselben Punktes zur Zeit $t$ in Bezug auf ein im Raume festes Coordinatensystem, und es sei weitër:

$$
\begin{aligned}
& \xi=\alpha+\alpha_{1} x+\alpha_{2} y+\alpha_{3} z, \\
& \eta=\beta+\beta_{1} x+\beta_{2} y+\beta_{3} z, \\
& \zeta=\gamma+\gamma_{1} x+\gamma_{2} y+\gamma_{3} z .
\end{aligned}
$$

Die 12 Grössen $\alpha, \beta, \gamma$ sind dann Functionen der Zeit, um deren Bestimmung es sich handelt. Man setze ferner:

$$
\begin{aligned}
& u=\alpha_{1} \frac{d \alpha}{d t}+\beta_{1} \frac{d \beta}{d t}+\gamma_{1} \frac{d \gamma}{d t} \\
& v=\alpha_{2} \frac{d \alpha}{d t}+\beta_{2} \frac{d \beta}{d t}+\gamma_{2} \frac{d \gamma}{d t} \\
& w=\alpha_{3} \frac{d \alpha}{d t}+\beta_{3} \frac{d \beta}{d t}+\gamma_{3} \frac{d \gamma}{d t} \\
& p=\alpha_{2} \frac{d \alpha_{3}}{d t}+\beta_{2} \frac{d \beta_{3}}{d t}+\gamma_{2} \frac{d \gamma_{3}}{d t} \\
& q=\alpha_{3} \frac{d \alpha_{1}}{d t}+\beta_{3} \frac{d \beta_{1}}{d t}+\gamma_{3} \frac{d \gamma_{1}}{d t} \\
& r=\alpha_{1} \frac{d \alpha_{2}}{d t}+\beta_{1} \frac{d \beta_{3}}{d t}+\gamma_{1} \frac{d \gamma_{\beta}}{d t}
\end{aligned}
$$

d. h. man bezeichne durch $u, v$, w die Geschwindigkeiten des Anfangspunktes der $x, y$, z nach den Richtungen der $x, y, z$ und durch $p, q, r$ die Drehungsgeschwindigkeiten des Körpers um die Axen der $x, y, z$. Die lebendige Kraft 
des Körpers ist dann eine homogene Function zweiten Grades von $u, v, w$, $p, q, r$ mit constanten Coefficienten; es soll gezeigt werden, dass unter den genannten Voraussetzungen auch die lebendige Kraft der Flüssigkeit eine homogene Function zweiten Grades derselben Variabeln mit constanten Coefficienten ist.

Zu diesem Zwecke bezeichne man durch $x, y, z$ die Coordinaten eines Punktes des zur Zeit $t$ von der Flüssigkeit eingenommenen Raumes in Bezug auf das im Körper feste Coordinatensystem bei der Lage, welche dieses zur Zeit $t$ hat. Die Componenten der Geschwindigkeit, welche in diesem Punkte zur Zeit $t$ stattfindet, nach den Axen $\operatorname{der} x, y, z$ sind dann den gemachten Annahmen zufolge $\frac{\partial \varphi}{\partial x}, \frac{\partial \varphi}{\partial y}, \frac{\partial \varphi}{\partial z}$, wenn $\varphi$ das Geschwindigkeitspotential bedeutet, und es ist dieses eine in dem ganzen Gebiete von $x, y, z$ stetige und eindeutige Function dieser Variabeln, die der Differentialgleichung

$$
\frac{\partial^{2} \varphi}{\partial x^{2}}+\frac{\partial^{2} \varphi}{\partial y^{2}}+\frac{\partial^{2} \varphi}{\partial z^{2}}=0
$$

genügt. Bedeutet $\boldsymbol{N}$ die Normale eines Flächenelementes, so ist ferner für alke Elemente der Fläche, welche die Flüssigkeit im Unendlichen begrenzt, $\frac{\partial \varphi}{\partial N}=0$, und für jedes Element der Oberfläche des Körpers ist $\frac{\partial \varphi}{\partial N}=\operatorname{der}$ Componente der Geschwindigkeit des anliegenden Theiles des Körpers nach der Richtung von $N$.

Ist die Bewegung des Körpers, d. h. sind $u, v, v, p, q, r$ gegeben, so ist hiernach, einem bekannten Satze gemäss, $\varphi$ bis auf eine willkürliche additive Constante bestimmt. Es ist also $\varphi$, abgesehn von dieser Constanten, eine Function von $u, v, w, p, q, r$, und die lebendige Kraft der Flüssigkeit ist gleichfalls eine Function dieser sechs Variabeln. Um nachzuweisen, dass die lebendige Kraft der Flüssigkeit eine homogene Function zweiten Grades ist, ist nur zu zeigen, dass $\varphi$ einer linearen homogenen Function der mehrfach genannten Grössen gleichgesetzt werden kann. Die Richtigkeit der letzten Behauptung aber zeigt die folgende Ueberlegung.

Die Componenten der Geschwindigkeit eines Punktes des Körpers nach den Axen der $\xi, \eta, \zeta$ sind:

$$
\frac{d \xi}{d t}, \quad \frac{d \eta}{d t}, \quad \frac{d \zeta}{d t},
$$

also die nach den Axen der $x, y, z$ : 
240 Kirchhoff, über die Bewegung eines Rotationskörpers in einer Flüssigkeit.

$$
\begin{aligned}
& \alpha_{1} \frac{d \xi}{d t}+\beta_{1} \frac{d \eta}{d t}+\gamma_{1} \frac{d \zeta}{d t} \\
& \alpha_{2} \frac{d \xi}{d t}+\beta_{2} \frac{d \eta}{d t}+\gamma_{2} \frac{d \zeta}{d t} \\
& \alpha_{3} \frac{d \xi}{d t}+\beta_{3} \frac{d \eta}{d t}+\gamma_{3} \frac{d \zeta}{d t}
\end{aligned}
$$

oder

$$
\text { (2.) }\left\{\begin{array}{c}
u+r y-q z, \\
v+p z-r x, \\
w+q x-p y,
\end{array}\right.
$$

wie aus den Gleichungen folgt:

$$
\begin{aligned}
& \frac{d \xi}{d t}=\frac{d \alpha}{d t}+\frac{d \alpha_{1}}{d t} x+\frac{d \alpha_{2}}{d t} y+\frac{d \alpha_{3}}{d t} z, \\
& \frac{d \eta}{d t}=\frac{d \beta}{d t}+\frac{d \beta_{1}}{d t} x+\frac{d \beta_{2}}{d t} y+\frac{d \beta_{3}}{d t} z, \\
& \frac{d \zeta}{d t}=\frac{d \gamma}{d t}+\frac{d \gamma_{1}}{d t} x+\frac{d \gamma_{2}}{d t} y+\frac{d \gamma_{3}}{d t} z
\end{aligned}
$$

in Verbindung mit den Definitionsgleichungen von $u, v, w, p, q, r$ und den Relationen, die zwischen $\alpha_{1}, \beta_{1}, \gamma_{1}, \alpha_{2}, \beta_{2}, \gamma_{2}, \alpha_{3}, \beta_{3}, \gamma_{3}$ bestehen. Die Bedingung, der $\varphi$ an der Oberfläche des Körpers zu genügen hat, ist hiernach : $\frac{d \varphi}{d N}=(u+r y-q z) \cos (N, x)+(v+p z-r x) \cos (N, y)+(w+q x-p y) \cos (N, z)$.

Man erfüllt daher alle für $\varphi$ aufgestellten Forderungen, indem man

(3.) $\varphi=u \varphi_{1}+v \varphi_{2}+v \varphi_{3}+p \varphi_{4}+q \varphi_{5}+r \varphi_{6}$

setzt und die Functionen $\varphi_{1}, \varphi_{2}, \ldots, \varphi_{6}$ so bestimmt, dass jede von ihnen den für $\varphi$ angegebenen Bedingungen genügt mit Ausnahme derjenigen, die sich auf die Oberfläche des Körpers bezieht, und dass für diese'Oberfläche:

$$
\text { (4.) } \quad \begin{cases}\frac{\partial \varphi_{1}}{\partial N}=\cos (N, x), & \frac{\partial \varphi_{4}}{\partial N}=z \cos (N, y)-y \cos (N, z) \\ \frac{\partial \varphi_{2}}{\partial N}=\cos (N, y), & \frac{\partial \varphi_{3}}{\partial N}=x \cos (N, z)-z \cos (N, x) \\ \frac{\partial \varphi_{3}}{\partial N}=\cos (N, z), & \frac{\partial \varphi_{6}}{\partial N}=y \cos (N, x)-x \cos (N, y)\end{cases}
$$

ist. Diese Functionen $\varphi_{1}, \varphi_{2}, \ldots \varphi_{6}$ sind unabhăngig von $u, v, v, p, q, r$; es kann daher $\varphi$ einer linearen homogenen Function dieser Grössen gleichgesetzt werden. Ferner sind $\varphi_{1}, \varphi_{2}, \ldots \varphi_{6}$ ausser von $x, y, z$ ausschliess- 
lich von der Gesłalt des Körpers abhängig. Daraus folgt, dass die lebendige Kraft der Flüssigkeit eine homogene Function zweiten Grades von $u, v, w$, $p, q, r$ ist, deren Coefficienten durch die Gestalt des Körpers und die Dichtigkeit der Flüssigkeit bestimmt sind.

\section{\$. 2 .}

Nach den gemachten Auseinandersetzungen ist die in Gleichung. (1.) mil $T$ bezeichnete lebendige Kraft des Körpers und der Flüssigkeit eine homogene Function zweiten Grades von $u, v, w, p, q, r$ mit constanten Coefficienten; nach der Annahme ist $\Omega$ eine Function der Coordinaten $\alpha, \beta, \gamma$ und der Cosinus $\alpha_{1}, \beta_{1}, \gamma_{1}, \alpha_{2}, \beta_{2}, \gamma_{2}, \alpha_{3}, \beta_{3}, \gamma_{3}$. Zwischen den 18 Variabeln, von welchen hiernach $\Omega+T$ abhängt, bestehn 12 Bedingungsgleichungen, die 6 Definitionsgleichungen für $u, v, w, p, q, r$ nämlich und die 6 Relationen zwischen den 9 Cosinus $\alpha_{1}, \beta_{1}, \ldots$ Nach den Regeln der Variationsrechnung hat man, um die Gleichung (1.) zu entwickeln, zu $\Omega+T$ hinzuzufügen die mit unbestimmten Factoren multiplicirten Ausdrücke, welche den Bedingungsgleichungen zufolge gleich Null sein sollen, und, wenn $S$ die so erhaltene Sumıne bedeutet, die Gleichung

$$
0=\delta \int S d t
$$

unter der Voraussetzung zu entwickeln, dass jene Variabeln und diese Factoren unabhängige Functionen von $t$ sind. Die 18 Gleichungen, die man aus der Gleichung

$$
\frac{\partial S}{\partial s}=\frac{d}{d t}\left(\frac{\partial S}{\partial \frac{d s}{d t}}\right)
$$

erhält, wenn man hier für $s$ der Reihe nach jene 18 Variabeln setzt, bilden in Verbindung mit den 12 Bedingungsgleichungen die gesuchte Entwicklung.

Die folgende Zusammenstellung giebt die Bedingungsgleichungen an und die entsprechenden Factoren, die eingeführt werden sollen.

Bedingungsgleichungen.

$$
\begin{aligned}
& \alpha_{1} \frac{d \alpha}{d t}+\beta_{1} \frac{d \beta}{d t}+\gamma_{1} \frac{d \gamma}{d t}-u=0 \\
& \alpha_{2} \frac{d \alpha}{d t}+\beta_{2} \frac{d \beta}{d t}+\gamma_{2} \frac{d \gamma}{d t}-v=0 \\
& \alpha_{3} \frac{d \alpha}{d t}+\beta_{3} \frac{d \beta}{d t}+\gamma_{3} \frac{d \gamma}{d t}-w=0
\end{aligned}
$$

Journal für Mathematik Bd. LXXI. Heft 3.
Factoren.

$\boldsymbol{W}$ 
242 Kirchhoff, über die Bewegung eines Rotationskörpers in einer Fliissigkeit.

Bedingungsgleichungen.

Factoren.

$$
\begin{aligned}
& \alpha_{2} \frac{d \alpha_{3}}{d t}+\beta_{2} \frac{d \beta_{3}}{d t}+\gamma_{2} \frac{d \gamma_{3}}{d t}-p=0 \\
& \alpha_{3} \frac{d \alpha_{1}}{d t}+\beta_{3} \frac{d \beta_{1}}{d t}+\gamma_{3} \frac{d \gamma_{1}}{d t}-q=0 \\
& \alpha_{1} \frac{d \alpha_{2}}{d t}+\beta_{1} \frac{d \beta_{2}}{d t}+\gamma_{1} \frac{d \gamma_{2}}{d t}-r=0 \\
& \alpha_{1}^{2}+\beta_{1}^{2}+\gamma_{1}^{2}-1=0 \\
& \alpha_{2}^{2}+\beta_{2}^{2}+\gamma_{2}^{2}-1=0 \\
& \alpha_{3}^{2}+\beta_{3}^{2}+\gamma_{3}^{2}-1=0 \\
& \alpha_{2} \alpha_{3}+\beta_{2} \beta_{3}+\gamma_{2} \gamma_{3}=0 \\
& \alpha_{3} \alpha_{1}+\beta_{3} \beta_{1}+\gamma_{3} \gamma_{1}=0 \\
& \alpha_{1} \alpha_{2}+\beta_{1} \beta_{2}+\gamma_{1} \gamma_{2}=0
\end{aligned}
$$

Daraus folgen diese 18 Gleichungen:

$$
\begin{gathered}
\frac{\partial T}{\partial u}=U, \quad \frac{\partial T}{\partial p}=P, \\
\frac{\partial T}{\partial v}=V, \quad \frac{\partial T}{\partial q}=Q \\
\frac{\partial T}{\partial w}=W, \quad \frac{\partial T}{\partial r}=R, \\
\frac{\partial \Omega}{\partial \alpha}=\frac{d}{d t}\left(\alpha_{1} U+\alpha_{2} V+\alpha_{3} W\right) \\
\frac{\partial \Omega}{\partial \beta}=\frac{d}{d t}\left(\beta_{1} U+\beta_{2} V+\beta_{3} W\right) \\
\frac{\partial \Omega}{\partial \gamma}=\frac{d}{d t}\left(\gamma_{1} U+\gamma_{2} V+\gamma_{3} W\right), \\
\frac{\partial \Omega}{\partial \alpha_{1}}+U \frac{d \alpha}{d t}+R \frac{d \alpha_{2}}{d t}+\lambda_{11} \alpha_{1}+\lambda_{12} \alpha_{2}+\lambda_{13} \alpha_{3}=\frac{d}{d t}\left(\alpha_{3} Q\right), \\
\frac{\partial \Omega}{\partial \beta_{1}}+U \frac{d \beta}{d t}+R \frac{d \beta_{2}}{d t}+\lambda_{12} \beta_{1}+\lambda_{12} \beta_{2}+\lambda_{13} \beta_{3}=\frac{d}{d t}\left(\beta_{3} Q\right), \\
\frac{\partial \Omega}{\partial \gamma_{1}}+U \frac{d \gamma}{d t}+R \frac{d \gamma_{2}}{d t}+\lambda_{11} \gamma_{1}+\lambda_{12} \gamma_{2}+\lambda_{13} \gamma_{3}=\frac{d}{d t}\left(\gamma_{3} Q\right), \\
\frac{\partial \Omega}{\partial \alpha_{2}}+V \frac{d \alpha}{d t}+P \frac{d \alpha_{3}}{d t}+\lambda_{21} \alpha_{1}+\lambda_{22} \alpha_{2}+\lambda_{23} \alpha_{3}=\frac{d}{d t}\left(\alpha_{1} R\right), \\
\frac{\partial \Omega}{\partial \beta_{2}}+V \frac{d \beta}{d t}+P \frac{d \beta_{3}}{d t}+\lambda_{21} \beta_{1}+\lambda_{22} \beta_{2}+\lambda_{23} \beta_{3}=\frac{d}{d t}\left(\beta_{1} R\right), \\
\frac{\partial \Omega}{\partial \gamma_{2}}+V \frac{d \gamma}{d t}+P \frac{d \gamma_{3}}{d t}+\lambda_{21} \gamma_{1}+\lambda_{22} \gamma_{2}+\lambda_{23} \gamma_{3}=\frac{d}{d t}\left(\gamma_{1} R\right)
\end{gathered}
$$


Kirchhoff, über die Bewegung eines Rotationskörpers in einer Flüssigkeit.

$$
\begin{aligned}
& \frac{\partial \Omega}{\partial \alpha_{3}}+W \frac{d \alpha}{d t}+Q \frac{d \alpha_{1}}{d t}+\lambda_{31} \alpha_{1}+\lambda_{32} \alpha_{2}+\lambda_{33} \alpha_{3}=\frac{d}{d t}\left(\alpha_{2} P\right), \\
& \frac{\partial \Omega}{\partial \beta_{3}}+W \frac{d \beta}{d t}+Q \frac{d \beta_{1}}{d t}+\lambda_{31} \beta_{1}+\lambda_{32} \beta_{2}+\lambda_{33} \beta_{3}=\frac{d}{d t}\left(\beta_{2} P\right), \\
& \frac{\partial \Omega}{\partial \gamma_{3}}+W \frac{d \gamma}{d t}+Q \frac{d \gamma_{1}}{d t}+\lambda_{31} \gamma_{1}+\lambda_{32} \gamma_{2}+\lambda_{33} \gamma_{3}=\frac{d}{d t}\left(\gamma_{2} P\right) .
\end{aligned}
$$

Die Werthe von $U, V, W, P, Q, R$, welche die 6 ersten Gleichungen geben, substituire man in die übrigen; die 3 folgenden erhalten dann unmittelbar eine zum weitern Gebrauche geeignete Form, während aus den 9 letzten noch die 6 Grössen $\lambda$ eliminirt werden müssen. Jene 3 Gleichungen werden:

$$
\left(5^{a} .\right)\left\{\begin{array}{l}
\frac{d}{d t}\left(\alpha_{1} \frac{\partial \boldsymbol{T}}{\partial u}+\alpha_{2} \frac{\partial \boldsymbol{T}}{\partial v}+\alpha_{3} \frac{\partial \boldsymbol{T}}{\partial w}\right)=\frac{\partial \Omega}{\partial \boldsymbol{\alpha}}, \\
\frac{d}{d t}\left(\beta_{1} \frac{\partial \boldsymbol{T}}{\partial u}+\beta_{2} \frac{\partial \boldsymbol{T}}{\partial v}+\beta_{3} \frac{\partial \boldsymbol{T}}{\partial w}\right)=\frac{\partial \Omega}{\partial \beta}, \\
\frac{d}{d t}\left(\gamma_{1} \frac{\partial \boldsymbol{T}}{\partial u}+\gamma_{2} \frac{\partial \boldsymbol{T}}{\partial v}+\gamma_{3} \frac{\partial \boldsymbol{T}}{\partial w}\right)=\frac{\partial \Omega}{\partial \gamma} .
\end{array}\right.
$$

Die Grössen, die auf der rechten Seite der Gleichheitszeichen hier stehen, sind die Summen der Componenten der auf den Körper wirkenden Kräfte nach den Axen der $\xi, \eta$, $\zeta$.

Multiplicirt man die Gleichungen $\left(5^{a}\right.$.)

$$
\begin{aligned}
& \text { mit } \alpha_{1} \text { oder mit } \alpha_{2} \text { oder mit } \alpha_{3} \\
& -\beta_{1}--\beta_{2}--\beta_{3} \\
& -\gamma_{1}--\gamma_{2}--\gamma_{3}
\end{aligned}
$$

und addirt sie jedesmal, so erhält man bei Rücksicht auf die Gleichungen, die zwischen diesen 9 Cosinus und den Grössen $p, q, r$ bestehen:

$$
\left(\boldsymbol{5}^{b} .\right) \quad\left\{\begin{array}{l}
\frac{d}{d t} \frac{\partial \boldsymbol{T}}{\partial u}=q \frac{\partial \boldsymbol{T}}{\partial w}-r \frac{\partial \boldsymbol{T}}{\partial v}+\alpha_{1} \frac{\partial \Omega}{\partial \alpha}+\beta_{1} \frac{\partial \boldsymbol{\Omega}}{\partial \beta}+\gamma_{1} \frac{\partial \boldsymbol{\Omega}}{\partial \gamma} \\
\frac{d}{d t} \frac{\partial \boldsymbol{T}}{\partial v}=r \frac{\partial \boldsymbol{T}}{\partial u}-p \frac{\partial \boldsymbol{T}}{\partial w}+\alpha_{2} \frac{\partial \Omega}{\partial \alpha}+\beta_{2} \frac{\partial \Omega}{\partial \beta}+\gamma_{2} \frac{\partial \Omega}{\partial \gamma} \\
\frac{d}{d t} \frac{\partial \boldsymbol{T}}{\partial w}=p \frac{\partial \boldsymbol{T}}{\partial v}-q \frac{\partial T}{\partial u}+\alpha_{3} \frac{\partial \Omega}{\partial \alpha}+\beta_{3} \frac{\partial \Omega}{\partial \beta}+\gamma_{3} \frac{\partial \boldsymbol{\Omega}}{\partial \gamma}
\end{array}\right.
$$

Die erwähnte Elimination der Grössen $\lambda$ aus den 9 Gleichungen, in denen diese vorkommen, kann man bewirken, indem man die Gleichungen mit 0 oder mit $-\gamma_{1}$ oder mit $\beta_{1}$

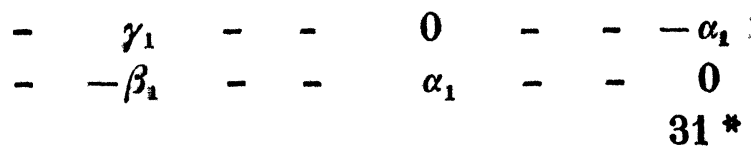


244 Kirchhoff, ïber die Bewegung eines Rotationskörpers in einer Flüssigkeit.

$$
\begin{array}{cccccccc}
\text { mit } & 0 & \text { oder } & \text { mit } & -\gamma_{2} & \text { oder } & \text { mit } & \beta_{2} \\
- & \gamma_{2} & - & - & 0 & - & - & -\alpha_{2} \\
- & -\beta_{2} & - & - & \alpha_{2} & - & - & 0 \\
- & 0 & - & - & -\gamma_{3} & - & - & \beta_{3} \\
- & \gamma_{3} & - & - & 0 & - & - & -\alpha_{3} \\
- & -\beta_{3} & - & - & \alpha_{3} & - & - & 0
\end{array}
$$

multiplicirt und jedesmal addirt. Setzt man der Kürze wegen

$$
\begin{aligned}
& \boldsymbol{M}_{\xi}=\gamma \frac{\partial \boldsymbol{\Omega}}{\partial \beta}-\beta \frac{\partial \boldsymbol{\Omega}}{\partial \gamma}+\gamma_{1} \frac{\partial \boldsymbol{\Omega}}{\partial \beta_{1}}-\beta_{1} \frac{\partial \boldsymbol{\Omega}}{\partial \gamma_{1}}+\gamma_{2} \frac{\partial \boldsymbol{\Omega}}{\partial \beta_{2}}-\beta_{2} \frac{\partial \boldsymbol{\Omega}}{\partial \gamma_{2}}+\gamma_{3} \frac{\partial \boldsymbol{\Omega}}{\partial \beta_{3}}-\beta_{3} \frac{\partial \boldsymbol{\Omega}}{\partial \gamma_{3}}, \\
& \boldsymbol{M}_{\eta}=\alpha \frac{\partial \boldsymbol{\Omega}}{\partial \gamma}-\gamma \frac{\partial \boldsymbol{\Omega}}{\partial \alpha}+\alpha_{1} \frac{\partial \boldsymbol{\Omega}}{\partial \gamma_{1}}-\gamma_{1} \frac{\partial \boldsymbol{\Omega}}{\partial \alpha_{1}}+\alpha_{2} \frac{\partial \boldsymbol{\Omega}}{\partial \gamma_{2}}-\gamma_{2} \frac{\partial \boldsymbol{\Omega}}{\partial \alpha_{2}}+\alpha_{3} \frac{\partial \boldsymbol{\Omega}}{\partial \gamma_{3}}-\gamma_{3} \frac{\partial \boldsymbol{\Omega}}{\partial \alpha_{3}}, \\
& \boldsymbol{M}_{\zeta}=\beta \frac{\partial \boldsymbol{\Omega}}{\partial \alpha}-\alpha \frac{\partial \boldsymbol{\Omega}}{\partial \boldsymbol{\beta}}+\boldsymbol{\beta}_{1} \frac{\partial \boldsymbol{\Omega}}{\partial \alpha_{1}}-\alpha_{1} \frac{\partial \boldsymbol{\Omega}}{\partial \boldsymbol{\beta}_{1}}+\beta_{2} \frac{\partial \boldsymbol{\Omega}}{\partial \alpha_{2}}-\alpha_{2} \frac{\partial \boldsymbol{\Omega}}{\partial \boldsymbol{\beta}_{2}}+\beta_{3} \frac{\partial \boldsymbol{\Omega}}{\partial \alpha_{3}}-\alpha_{3} \frac{\partial \boldsymbol{\Omega}}{\partial \boldsymbol{\beta}_{3}},
\end{aligned}
$$

d. h. bezeichnet man durch $M_{\xi}, M_{\eta}, M_{\zeta}$ die Drehungsmomente der auf den Körper wirkenden Kräfte in Bezug auf die Axen der $\xi, \eta, \zeta$; nimmt man ferner an, dass die Axen der $x, y, z$ durch Drehung den Axen der $\xi, \eta$, $\zeta$ parallel gemacht werden können, so dass

$$
\begin{array}{lll}
\alpha_{1}=\beta_{2} \gamma_{3}-\beta_{3} \gamma_{2}, & \beta_{1}=\gamma_{2} \alpha_{3}-\gamma_{3} \alpha_{2}, & \gamma_{1}=\alpha_{2} \beta_{3}-\alpha_{3} \beta_{2}, \\
\alpha_{2}=\beta_{3} \gamma_{1}-\beta_{1} \gamma_{3}, & \beta_{2}=\gamma_{3} \alpha_{1}-\gamma_{1} \alpha_{3}, & \gamma_{2}=\alpha_{3} \beta_{1}-\alpha_{1} \beta_{3}, \\
\alpha_{3}=\beta_{1} \gamma_{2}-\beta_{2} \gamma_{1}, & \beta_{3}=\gamma_{1} \alpha_{2}-\gamma_{2} \alpha_{1}, & \gamma_{3}=\alpha_{1} \beta_{2}-\alpha_{2} \beta_{1}
\end{array}
$$

ist, so erhält man auf dem angegebenen Wege nach einigen Transformationen und bei Rücksicht auf die Gleichungen $\left(5^{a}.\right)$ :

$\left(6^{a}.\right)$

$$
\left\{\begin{array}{c}
\frac{d}{d t}\left\{\begin{array}{cc}
\left(\beta_{1} \gamma-\gamma_{1} \beta\right) \frac{\partial T}{\partial u}+\left(\beta_{2} \gamma-\gamma_{2} \beta\right) \frac{\partial T}{\partial v}+\left(\beta_{3} \gamma-\gamma_{3} \beta\right) \frac{\partial T}{\partial w} \\
+\alpha_{1} \frac{\partial T}{\partial p}+\alpha_{2} \frac{\partial T}{\partial q} & +\alpha_{3} \frac{\partial T}{\partial r}
\end{array}\right\}=M_{\xi}, \\
\frac{d}{d t}\left\{\begin{array}{cc}
\left(\gamma_{1} \alpha-\alpha_{1} \gamma\right) \frac{\partial T}{\partial u}+\left(\gamma_{2} \alpha-\alpha_{2} \gamma\right) \frac{\partial T}{\partial v}+\left(\gamma_{3} \alpha-\alpha_{3} \gamma\right) \frac{\partial T}{\partial w} \\
+\beta_{1} \frac{\partial T}{\partial p}+\beta_{2} \frac{\partial T}{\partial q} & +\beta_{3} \frac{\partial T}{\partial r}
\end{array}\right\}=M_{\eta}, \\
\frac{d}{d t}\left\{\begin{array}{c}
\left(\alpha_{1} \beta-\beta_{1} \alpha\right) \frac{\partial T}{\partial u}+\left(\alpha_{2} \beta-\beta_{2} \alpha\right) \frac{\partial T}{\partial v}+\left(\alpha_{3} \beta-\beta_{3} \alpha\right) \frac{\partial T}{\partial w} \\
+\gamma_{1} \frac{\partial T}{\partial p}+\gamma_{2} \frac{\partial T}{\partial q}
\end{array}\right\}=M_{\zeta} .
\end{array}\right.
$$

Die Elimination der Grössen $\lambda$ aus den 9 Gleichungen, in denen sie vorkommen, lässt sich auch dadurch erreichen, dass man diese Gleichungen 
Kirchhoff, über die Bewegung eines Rotationskörpers in einer Flüssigkeit. 245

$$
\begin{aligned}
& \text { mit } \quad 0 \text { oder mit }-\alpha_{3} \text { oder mit } \alpha_{2} \\
& \text { - } 0--\beta_{3}--\beta_{2} \\
& -0=-\gamma_{3}--\gamma_{2} \\
& -\alpha_{3}--0--\alpha_{1} \\
& -\beta_{3}--0---\beta_{1} \\
& -\gamma_{3}--0---\gamma_{1} \\
& -\quad-\alpha_{2}--\alpha_{1}-\quad-0 \\
& --\beta_{2}--\beta_{1}--0 \\
& --\gamma_{2}--\gamma_{1}--0
\end{aligned}
$$

multiplicirt und jedesmal addirt; dadurch erhält man:

$$
\left(6^{b} .\right)\left\{\begin{aligned}
\frac{d}{d t} \frac{\partial T}{\partial p}= & v \frac{\partial T}{\partial w}-w \frac{\partial T}{\partial v}+q \frac{\partial T}{\partial r}-r \frac{\partial T}{\partial q} \\
& +\alpha_{2} \frac{\partial \Omega}{\partial \alpha_{3}}-\sigma_{3} \frac{\partial \Omega}{\partial \alpha_{2}}+\beta_{2} \frac{\partial \Omega}{\partial \beta_{3}}-\beta_{3} \frac{\partial \Omega}{\partial \beta_{2}}+\gamma_{2} \frac{\partial \Omega}{\partial \gamma_{3}}-\gamma_{3} \frac{\partial \Omega}{\partial \gamma_{2}} \\
\frac{d}{d t} \frac{\partial T}{\partial q}= & w \frac{\partial T}{\partial u}-u \frac{\partial T}{\partial w}+r \frac{\partial T}{\partial p}-p \frac{\partial T}{\partial r} \\
& +\alpha_{3} \frac{\partial \Omega}{\partial \alpha_{1}}-\alpha_{1} \frac{\partial \Omega}{\partial \alpha_{3}}+\beta_{3} \frac{\partial \Omega}{\partial \beta_{1}}-\beta_{1} \frac{\partial \Omega}{\partial \beta_{3}}+\gamma_{3} \frac{\partial \Omega}{\partial \gamma_{1}}-\gamma_{1} \frac{\partial \Omega}{\partial \gamma_{3}} \\
\frac{d}{d t} \frac{\partial T}{\partial r}= & u \frac{\partial T}{\partial v}-v \frac{\partial T}{\partial u}+p \frac{\partial T}{\partial q}-q \frac{\partial T}{\partial p} \\
& +\alpha_{1} \frac{\partial \Omega}{\partial \alpha_{2}}-\alpha_{2} \frac{\partial \Omega}{\partial \alpha_{1}}+\beta_{1} \frac{\partial \Omega}{\partial \beta_{2}}-\beta_{2} \frac{\partial \Omega}{\partial \beta_{1}}+\gamma_{1} \frac{\partial \Omega}{\partial \gamma_{2}}-\gamma_{2} \frac{\partial \Omega}{\partial \gamma_{1}}
\end{aligned}\right.
$$

\section{§. 3.}

Es soll jetzt die Annahme eingeführt werden, dass keine Kräfte auf den Körper wirken, das Potential $\Omega$ also gleich Null gesetzt werden kann. Die Gleichungen $\left(5^{b}.\right)$ und $\left(6^{b}.\right)$ nehmen dann die folgende, einfache Gestalt an:

$$
\left\{\begin{array}{l}
\frac{d}{d t} \frac{\partial T}{\partial u}=q \frac{\partial T}{\partial w}-r \frac{\partial T}{\partial v}, \\
\frac{d}{d t} \frac{\partial T}{\partial v}=r \frac{\partial T}{\partial u}-p \frac{\partial T}{\partial v}, \\
\frac{d}{d t} \frac{\partial T}{\partial w}=p \frac{\partial T}{\partial v}-q \frac{\partial T}{\partial u}, \\
\frac{d}{d t} \frac{\partial T}{\partial p}=v \frac{\partial T}{\partial w}-v \frac{\partial T}{\partial v}+q \frac{\partial T}{\partial r}-r \frac{\partial T}{\partial q}, \\
\frac{d}{d t} \frac{\partial T}{\partial q}=v \frac{\partial T}{\partial u}-u \frac{\partial T}{\partial w}+r \frac{\partial T}{\partial p}-p \frac{\partial T}{\partial r} \\
\frac{d}{d t} \frac{\partial T}{\partial v}=u \frac{\partial T}{\partial v}-v \frac{\partial T}{\partial u}+p \frac{\partial T}{\partial q}-q \frac{\partial T}{\partial p}
\end{array}\right.
$$


246 Kirchhoff, über die Bewegung eines Rotationskörpers in einer Flüssigkeit.

Man findet 3 allgemeine Integrale dieser Gleichungen, indem man sie

$$
\begin{aligned}
& \text { mit } u \text { oder mit } \frac{\partial T}{\partial u} \text { oder mit } \frac{\partial T}{\partial p} \\
& -v-\quad-\frac{\partial \boldsymbol{T}}{\partial \boldsymbol{v}}--\frac{\partial \boldsymbol{T}}{\partial \boldsymbol{q}} \\
& -w--\frac{\partial T}{\partial \boldsymbol{w}}--\frac{\partial \boldsymbol{T}}{\partial \boldsymbol{r}} \\
& -p-\quad 0 \quad-\quad-\frac{\partial T}{\partial u} \\
& -\boldsymbol{q}-\mathbf{0}--\frac{\partial T}{\partial v} \\
& -r-\quad 0--\frac{\partial \boldsymbol{T}}{\partial \boldsymbol{w}}
\end{aligned}
$$

multiplicirt und jedesmal addirt.

Erwägt man, dass

$$
2 \boldsymbol{T}=u \frac{\partial T}{\partial u}+v \frac{\partial T}{\partial v}+w \frac{\partial T}{\partial w}+p \frac{\partial T}{\partial p}+q \frac{\partial T}{\partial q}+r \frac{\partial T}{\partial r}
$$

und

$$
\frac{d T}{d t}=\frac{\partial T}{\partial u} \frac{d u}{d t}+\frac{\partial T}{\partial v} \frac{d v}{d t}+\frac{\partial T}{\partial w} \frac{d w}{d t}+\frac{\partial T}{\partial p} \frac{d p}{d t}+\frac{\partial T}{\partial q} \frac{d q}{d t}+\frac{\partial T}{\partial r} \frac{d r}{d t}
$$

ist, so ergiebt das erste Faktorensystem:

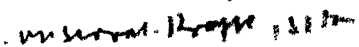

. $\quad k=0$

unst.

$$
2 T=L ; \quad(\ln m+n)^{2}
$$

die beiden andern ergeben:

$$
\begin{array}{r}
\left(\frac{\partial T}{\partial u}\right)^{2}+\left(\frac{\partial T}{\partial v}\right)^{2}+\left(\frac{\partial T}{\partial v}\right)^{2}=M, \\
\frac{\partial T}{\partial u} \frac{\partial T}{\partial p}+\frac{\partial T}{\partial v} \frac{\partial T}{\partial q}+\frac{\partial T}{\partial v} \frac{\partial T}{\partial r}=N
\end{array}
$$

wo $L, M, N$ willkürliche Constanten bedeuten.

6 andere Integralgleichungen des vorliegenden Problems erhält man aus den Gleichungen $\left(5^{a}.\right)$ und $\left(6^{a}.\right)$. Setzt man in diesen der eingeführten Voraussetzung gemäss

$$
\frac{\partial \boldsymbol{\Omega}}{\partial \alpha}, \frac{\partial \boldsymbol{\Omega}}{\partial \boldsymbol{\beta}}, \frac{\partial \boldsymbol{\Omega}}{\partial \gamma}, \boldsymbol{M}_{\xi}, \quad \boldsymbol{M}_{\eta}, \boldsymbol{M}_{\zeta}
$$

gleich Null, so folgt aus ihnen: 


$$
\begin{aligned}
& \text { (9.) }\left\{\begin{array}{l}
\alpha_{1} \frac{\partial T}{\partial u}+\alpha_{2} \frac{\partial T}{\partial v}+\alpha_{3} \frac{\partial T}{\partial w}=\mathbf{A} \\
\beta_{1} \frac{\partial T}{\partial u}+\beta_{2} \frac{\partial T}{\partial v}+\beta_{3} \frac{\partial T}{\partial w}=\mathbf{B} \\
\gamma_{1} \frac{\partial T}{\partial u}+\gamma_{2} \frac{\partial T}{\partial v}+\gamma_{3} \frac{\partial T}{\partial w}=\mathbf{C}
\end{array}\right. \\
& \text { (10.) }\left\{\begin{array}{l}
\alpha_{1} \frac{\partial T}{\partial p}+\alpha_{2} \frac{\partial T}{\partial q}+\alpha_{3} \frac{\partial T}{\partial r}=A+\beta \mathrm{C}-\gamma \mathrm{B} \\
\beta_{1} \frac{\partial T}{\partial p}+\beta_{2} \frac{\partial T}{\partial q}+\beta_{3} \frac{\partial T}{\partial r}=B+\gamma \mathrm{A}-\alpha \mathrm{C} \\
\gamma_{1} \frac{\partial T}{\partial p}+\gamma_{2} \frac{\partial T}{\partial q}+\gamma_{3} \frac{\partial T}{\partial r}=\Gamma+\alpha \mathrm{B}-\beta \mathrm{A}
\end{array}\right.
\end{aligned}
$$

wo $\mathrm{A}, \mathrm{B}, \mathrm{C}, A, B, \boldsymbol{I}$ ' willkürliche Constanten bezeichnen.

Die beiden letzten der Gleichungen (8.) sind Folgen der Gleichungen (9.) und (10.), und die Constanten $M$ und $N$ sind durch die Constanten $\mathrm{A}, \mathrm{B}, \mathrm{C}, A, B, I$ ausdrückbar. Quadrirt man nämlich die Gleichungen' (9.) und addirt sie, multiplicirt man dann die Gleichungen (9.) mit den Gleichungen (10.) und addirt sie wieder, so erhält man bei Rücksicht auf die Gleichungen (8.):

$$
\text { (11.) } \quad\left\{\begin{array}{l}
\mathrm{A}^{2}+\mathrm{B}^{2}+\mathrm{C}^{2}=M, \\
\mathrm{~A} A+\mathrm{B} B+\mathrm{C} I=N .
\end{array}\right.
$$

\section{S. 4.}

Bevor vereinfachende Voraussetzungen in Bezug auf die Gestalt des Körpers und die Vertheilung der Masse in ihm eingeführt werden, möge eine particuläre Lösung der vorliegenden Differentialgleichungen angeführt werden, welche unter denselben Annahmen gilt, als die im vorigen Paragraphen abgeleiteten Gleichungen.

Die Gleichungen (7.) erfüllt man, wenn man $p=0, q=0, r=0$ und $u, v, w$ gleich Constanten setzt, deren Verhältnisse man passend bestimmt, nämlich so, dass

$$
u: v: w=\frac{\partial T}{\partial u}: \frac{\partial T}{\partial v}: \frac{\partial T}{\partial w}
$$

ist. Erwägt man, dass, wenn $p, q, r$ verschwinden, $T$ eine homogene Function zweiten Grades von $u, v, v$ wird, und zwar eine, die stets positiv bleibt, so sieht man; dass die Bestimmung der Verhältnisse u:ø:w übereinkommt 
248 Kirchhoff, iiber die Bewegung eines Rotationskörpers in einer Fliissigkeit.

mit der Bestinmung der Hauptaxen eines gewissen Ellipsoids. Es folgt daraus mit Leichtigkeit, dass es für jeden Körper 3, und im Allgemeinen nur 3, auf einander senkrechte Richtungen giebt, in denen er ohne sich zu drehen in der Flüssigkeit fortschreiten kann.

\section{S. 5 .}

Die Zahl der Constanten, die in dem Ausdrucke. von $T$ vorkommen, ist im Allgemeinen 21; es soll jetzt untersucht werden, wie diese Zahl sich verringert, wenn der Körper gewisse Symmetrieen darbietet. Dabei soll zuerst der Theil von $\boldsymbol{T}$ ins Auge gefasst werden, den die lebendige Kraft der Flüssigkeit bildet. Das Doppelte dieser lebendigen Kraft setze man

$$
\begin{aligned}
&=a_{11} u^{2}+2 a_{12} u v+2 a_{13} u w+2 a_{14} u p+2 a_{15} u q+2 a_{16} u r \\
&+a_{22} v^{2}+2 a_{23} v w+2 a_{24} v p+2 a_{25} v q+2 a_{26} v r \\
&+a_{33} w^{2}+. . . \\
& .
\end{aligned}
$$

Dieser Ausdruck ist dann, wenn die Dichtigkeit der Flüssigkeit durch $\varrho$ bezeichnet wird,

$$
=\varphi \iint d x d y d z\left\{\left(\frac{\partial \varphi}{\partial x}\right)^{2}+\left(\frac{\partial \varphi}{\partial y}\right)^{2}+\left(\frac{\partial \varphi}{\partial z}\right)^{2}\right\},
$$

wo die Integration über den Raum auszudehnen ist, den die Flüssigkeit zur Zeit $t$ erfüllt, und $\varphi$ die in $\$ .1$ besprochene Function von $x, y, z$ bedeutet. Substituirt man hier für $\varphi$ den in Gleichung (3.) angegebenen Werth, so findet man für die Coefficienten $a$ die folgenden Ausdrücke:

$$
\begin{aligned}
& a_{11}=\varphi \iiint d x d y d z\left\{\left(\frac{\partial \varphi_{1}}{\partial x}\right)^{2}+\left(\frac{\partial \varphi_{1}}{\partial y}\right)^{2}+\left(\frac{\partial \varphi_{1}}{\partial z}\right)^{2}\right\}, \\
& a_{12}=\varphi \iiint d x d y d z\left\{\frac{\partial \varphi_{1}}{\partial x} \frac{\partial \boldsymbol{\varphi}_{2}}{\partial x}+\frac{\partial \varphi_{1}}{\partial y} \frac{\partial \varphi_{2}}{\partial y}+\frac{\partial \varphi_{1}}{\partial z} \frac{\partial \varphi_{2}}{\partial z}\right\},
\end{aligned}
$$

oder auch, wenn $d S$ ein Element der Oberfläche des Körpers und $\boldsymbol{N}$ die nach dem Innern der Flüssigkeit gerichtete Normale desselben bedeutet:

$$
\text { (12.) }\left\{\begin{array}{l}
a_{11}=-\rho \int d S \varphi_{1} \frac{\partial \varphi_{1}}{\partial N} \\
a_{12}=-\rho \int d S \varphi_{1} \frac{\partial \varphi_{2}}{\partial N} \\
. \cdot \cdot \cdot \cdot \cdot \cdot
\end{array}\right.
$$

Es werde nun angenommen, dass die Oberfläche des Körpers symmetrisch in Bezug auf die $x z$-Ebene ist, d.h. dass, wenn $x, y$, $z$ die Coor- 
dinaten eines Punktes derselben sind, sie auch den Punkt $x,-y, z$ enthält. Zwei Punkte, wie diese, sollen entsprechende Punkte genannt werden. In zwei entsprechenden Punkten der Oberfläche haben dann, den Gleichungen (4.) zufolge, $\frac{\partial \varphi_{1}}{\partial N}, \frac{\partial \varphi_{3}}{\partial N}, \frac{\partial \varphi_{5}}{\partial N}$ gleiche und $\frac{\partial \varphi_{2}}{\partial N}, \frac{\partial \varphi_{4}}{\partial N}, \frac{\partial \varphi_{6}}{\partial N}$ entgegengesetzte Werthe. Daraus folgt, dass in irgend zwei entsprechenden Punkten des von der Flüssigkeit erfüllten Raumes, also auch in zwei entsprechenden Punkten der Oberfläche des Körpers, $\varphi_{1}, \varphi_{3}, \varphi_{5}$ gleiche und $\varphi_{2}, \varphi_{4}, \varphi_{6}$, bei passender Bestimmung der in ihnen vorkommenden additiven Constanten, entgegengesetzte Werthe haben, und daraus ergiebt sich weiter, bei Rücksicht auf die Gleichungen (12.), dass diejenigen $a$ verschwinden, bei denen ein Index der Reihe 1, 3, 5, der andere der Reihe 2, 4, 6 angehört.

Die doppelte lebendige Kraft des Körpers ist, wenn $d m$ ein Element seiner Masse bezeiclnet, das die Coordinaten $x, y$, zesitzt, in Folge der Bedeutung der Ausdrücke (2.):

oder

$$
=\int d m\left\{(u+r y-q z)^{2}+(v+p z-r x)^{2}+(w+q x-p y)^{2}\right\}
$$

$$
\begin{gathered}
=\int d m\left\{u^{2}+v^{2}+w^{2}+\left(y^{2}+z^{2}\right) p^{2}+\left(z^{2}+x^{2}\right) q^{2}+\left(x^{2}+y^{2}\right) r^{2}\right. \\
+2 x(w q-v r)+2 y(u r-w p)+2 z(v p-u q) \\
-2 y z q r-2 z x r p-2 x y p q\}
\end{gathered}
$$

ist die Vertheilung der Masse symmetrisch zur $x z$-Ebene, so verschwinden hier diejenigen Glieder, welche die Factoren

$$
u r-w p, q r, \quad p q
$$

enthalten. Setzt man jenen Ausdruck allgemein

$$
\begin{array}{r}
=b_{11} u^{2}+2 b_{12} u v+2 b_{13} u w+2 b_{14} u p+\cdots \\
+b_{22} v^{2}+2 b_{23} v w+\cdots \cdots \cdots
\end{array}
$$

so verschwinden daher, sobald die Masse symmetrisch zur $x z$-Ebene vertheilt ist, diejenigen $b$; bei denen ein Index der Reihe 1, 3, 5, der andere der Reihe $2,4,6$ angehört.

Daraus folgt, dass, wenn man allgemein

$$
\begin{array}{r}
2 T=c_{11} u^{2}+2 c_{12} u v+2 c_{13} u v+2 c_{14} u p+\cdots \\
+c_{21} v^{2}+2 c_{23} v w+\cdots \cdots \cdots \cdots
\end{array}
$$


setzt, diejenigen $c$ gleich Null sind, bei denen ein Index der Reihe 1, 3, 5, der andere der Reihe 2, 4, 6 angehört, falls der Körper, sowohl in Bezug auf seine Gestalt, als in Bezug auf die Vertheilung der Masse in ihm, symmetrisch zur $x z$-Ebene ist.

Findet eine solche Symmetrie in Bezug auf die $y x$-Ebene oder die $z y$-Ebene statt, so treten an Stelle der Reihen 1, 3,5 und 2, 4, 6 die Reihen 2, 1, 6 und 3,5, 4 oder die Reihen 3,$2 ; 4$ und 1, 6, 5 .

Ist der Körper symmetrisch in Bezug auf die drei Coordinatenebenen, so enthält hiernach der Ausdruck von $2 T$ nur die Quadrate von $u, v, w, p, q, r$.

Es soll nun angenommen werden, dass der Körper der Gestalt und der Vertheilung der Masse nach ein Rotationskörper ist. dessen Axe mit der $x$-Axe zusammenfällt. Es findet dann Symmetrie in Bezug auf die $x y$-Ebene und in Bezug auf die $x z$-Ebene stalt, und daher ist

$$
\begin{gathered}
2 T=c_{11} u^{2}+c_{22} v^{2}+c_{33} w^{2}+c_{44} p^{2}+c_{55} q^{2}+c_{66} r^{2} \\
+2 c_{26} v r+2 c_{35} w q
\end{gathered}
$$

Zwischen den hier vorkommenden Constanten bestehen aber noch einige Beziehungen. Um diese zu finden, führe man neben dem System der $x, y$, noch ein zweites im Körper festes Coordinatensystem, das der $x^{\prime}, y^{\prime}, z^{\prime}$ ein; in Bezug auf dieses sollen $u^{\prime}, v^{\prime}, w^{\prime}, p^{\prime}, q^{\prime}, r^{\prime}, c_{11}^{\prime}, c_{12}^{\prime}, \ldots$ dieselbe Bedeutung haben, wie $u, v, w, p, q, r, c_{11}, c_{12}, \ldots$ in Bezug auf jenes. Die beiden Coordinatensysteme sollen denselben Anfangspunkt und dieselbe $x$-Axe haben, so dass, wenn $x, y, z$ und $x^{\prime}, y^{\prime}, z^{\prime}$, sich auf denselben Punkt beziehen:

$$
\begin{aligned}
& x=x^{\prime}, \\
& y=y^{\prime} \cos \vartheta+z^{\prime} \sin \vartheta, \\
& z=-y^{\prime} \sin \vartheta+z^{\prime} \cos \vartheta
\end{aligned}
$$

ist. Wegen der Bedeutung der Ausdrücke (2.) bestehen dann die Gleichungen:

$$
\begin{aligned}
& u+r y-\dot{q} z=u^{\prime}+r^{\prime} y^{\prime}-q^{\prime} z^{\prime}, \\
& v+p z-r x=\left(v^{\prime}+p^{\prime} z^{\prime}-r^{\prime} x^{\prime}\right) \cos \vartheta+\left(w^{\prime}+q^{\prime} x^{\prime}-p^{\prime} y^{\prime}\right) \sin \vartheta, \\
& w+q x-p y=-\left(v^{\prime}+p^{\prime} z^{\prime}-r^{\prime} x^{\prime}\right) \sin \vartheta+\left(w^{\prime}+q^{\prime} x^{\prime}-p^{\prime} y^{\prime}\right) \cos \vartheta,
\end{aligned}
$$

aus welchen folgt:

$$
\begin{aligned}
u & =u^{\prime}, & & p=p^{\prime}, \\
v & =v^{\prime} \cos \vartheta+w^{\prime} \sin \vartheta, & q & =q^{\prime} \cos \vartheta+r^{\prime} \sin \vartheta, \\
w & =-v^{\prime} \sin \vartheta+w^{\prime} \cos \vartheta, & & r=-q^{\prime} \sin \vartheta+r^{\prime} \cos \vartheta .
\end{aligned}
$$


Eine Symmetrie, wie in Bezug auf die $x y$ - und $x z$-Ebenen besteht auch in Bezug auf die $x^{\prime} y^{\prime}-$ und $x^{\prime} z^{\prime}-$ Ebenen; es ist daher auch

$$
\begin{gathered}
2 T=c_{11}^{\prime} u^{\prime 2}+c_{22}^{\prime} v^{\prime 2}+c_{33}^{\prime} w^{\prime 2}+c_{44}^{\prime} p^{\prime 3}+c_{55}^{\prime} q^{\prime 2}+c_{66}^{\prime} r^{\prime 2} \\
+2 c_{26}^{\prime} v^{\prime} r^{\prime}+2 c_{35}^{\prime} w^{\prime} q^{\prime} .
\end{gathered}
$$

Setzt man die beiden Ausdrücke von $2 T$ einander gleich und drückt die Grössen $u, v, w, p, q, r$ durch $u^{\prime}, v^{\prime}, w^{\prime}, p^{\prime}, q^{\prime}, r^{\prime}$ aus, so erhält man durch Vergleichung der Coefficienten der entsprechenden Glieder:

$$
c_{22}=c_{33}, \quad c_{55}=c_{6 i}, \quad c_{26}+c_{35}=0
$$

und Gleichungen, die aussagen, dass die Grössen $c^{\prime}$ den entsprechenden Grössen $c$ gleich sind. Hiernach ist

$$
2 T=c_{11} u^{2}+c_{22}\left(v^{2}+w^{2}\right)+c_{44} p^{2}+c_{55}\left(q^{2}+r^{2}\right)+2 c_{26}(v r-w q) .
$$

Dieser Ausdruck lässt noch eine Vereinfachung zu durch eine passende Wahl des Anfangspunktes der Coordinaten auf der $\boldsymbol{x}$-Axe. Um das zu zeigen, führe man, ähnlich wie oben, neben dem Coordinatensystem der $x,-y, z$ ein zweites, das der $x^{\prime}, y^{\prime}, z^{\prime}$ ein, das so gewählt sein soll, dass für jeden Punkt

$$
x=x^{\prime}+a, \quad y=y^{\prime}, \quad z=z^{\prime}
$$

ist. Bei einer ähnlichen Bezeichnung, wie sie oben gebraucht ist, hat man dann:

also

$$
\begin{aligned}
& u+r y-q z=u^{\prime}+r^{\prime} y^{\prime}-q^{\prime} z^{\prime}, \\
& v+p z-r x=v^{\prime}+p^{\prime} z^{\prime}-r^{\prime} x^{\prime}, \\
& w+q x-p y=v^{\prime}+q^{\prime} x^{\prime}-p^{\prime} y^{\prime},
\end{aligned}
$$

und

$$
\begin{aligned}
u & =u^{\prime}, & & p=p^{\prime}, \\
v & =v^{\prime}+r^{\prime} a, & & q=q^{\prime}, \\
w & =w^{\prime}-q^{\prime} a, & & r=r^{\prime}
\end{aligned}
$$

$$
\begin{aligned}
& c_{11} u^{2}+c_{22}\left(v^{2}+w^{2}\right)+c_{44} p^{2}+c_{55}\left(q^{2}+r^{2}\right)+2 c_{26}(v r-w q) \\
= & c_{11}^{\prime} u^{\prime 2}+c_{28}^{\prime}\left(v^{\prime 2}+w^{\prime 2}\right)+c_{44}^{\prime} p^{\prime 2}+c_{55}^{\prime}\left(q^{\prime 2}+r^{\prime 2}\right)+2 c_{26}^{\prime}\left(v^{\prime} r^{\prime}-w^{\prime} q^{\prime}\right),
\end{aligned}
$$

woraus folgt:

$$
\begin{aligned}
& c_{11}=c_{11}^{\prime}, \quad c_{22}=c_{22}^{\prime}, \quad c_{44}=c_{44}^{\prime}, \\
& c_{35}=c_{55}^{\prime}-2 c_{26}^{\prime} a+c_{22}^{\prime} a^{2}, \\
& c_{26}=c_{26}^{\prime}-c_{22}^{\prime} a .
\end{aligned}
$$

Hieraus geht hervor, dass, wenn der Anfangspunkt der $x^{\prime}$ beliebig gewählt ist, a so bestimmt werden kann, dass

$$
c_{26}=0, \ldots
$$


252 Kirchhoff, über die Bewegung eines Rotationskörpers in einer Flüssigkeit.

also

wird.

$$
2 T=c_{11} u^{2}+c_{22}\left(v^{2}+w^{2}\right)+c_{44} p^{2}+c_{55}\left(q^{2}+r^{2}\right)
$$

Es möge bemerkt werden, dass diese Gleichung, wie aus ihrer Herleitung ersichtlich ist, auch gilt, wenn der Körper kein Rotationskörper ist, sobald er nur symmetrisch ist in Bezug auf zwei oder mehr Paare aufeinander senkrechter Ebenen, die durch die $x$-Axe gehn; was z. B. bei einem homogenen, geraden Prisma oder einer solchen Pyramide von quadratischem oder regelmässig sechseckigem Querschnitt bei passender Wahl der $x$-Axe stattfindet. Auch für solche Fälle werden also die Folgerungen gelten, die an die Gleichung (13.) geknüpft werden sollen.

\section{\$. 6.}

Den durch die Gleichung (1.3.) bestimmten Werth von $T$ denke man sich nun in die Gleichungen (7.) substituirt. Die vierte von diesen wird dann:

$$
\frac{d p}{d t}=0 \quad \text { d. h. } \quad p=\text { const. }
$$

die andern werden:

$$
\left\{\begin{array}{l}
c_{11} \frac{d u}{d t}=-c_{22}(v r-w q) \\
c_{22} \frac{d v}{d t}=c_{11} u r-c_{22} w p \\
c_{22} \frac{d w}{d t}=-c_{11} u q+c_{22} v p \\
c_{55} \frac{d q}{d t}=\left(c_{11}-c_{22}\right) u v+\left(c_{44}-c_{55}\right) p r \\
c_{55} \frac{d r}{d t}=-\left(c_{11}-c_{22}\right) u v-\left(c_{44}-c_{55}\right) p q .
\end{array}\right.
$$

An Stelle der Variabeln $v, v, q, r$ sollen hier neue $s, \sigma, \varphi, \psi$ eingeführt werden, so dass

also

$$
\text { (15.) }\left\{\begin{array}{lll}
0=s \cos \varphi, & q=\sigma \cos (\varphi+\psi), \\
w=s \sin \varphi, & r=\sigma \sin (\varphi+\psi),
\end{array}\right.
$$

$$
\begin{gathered}
\sigma^{2}+w^{2}=b^{2}, q^{2}+r^{2}=\sigma^{2} \\
o q+v r=8 \sigma \cos \psi \\
o r-w q=\delta \sigma \sin \psi
\end{gathered}
$$


Kirchhoff, über die Bewegung eines Rotationskörpers in einer Flïssigkeit. 253

ist. Die erste der Gleichungen (14.) wird dann:

und, da

$$
\text { (16.) } \quad c_{11} \frac{d u}{d t}=-c_{22} s \sigma \sin \psi,
$$

$$
s^{2} d \varphi=-w d v+v d w
$$

ist, so folgt aus der zweiten und dritten:

$$
c_{22} \frac{d \varphi}{d t}=-c_{11} \frac{u \sigma}{s} \cos \psi+c_{22} p .
$$

Die drei Integralgleichungen (8.) werden:

$$
\left\{\begin{array}{l}
c_{11} u^{2}+c_{22} s^{2}+c_{44} p^{2}+c_{55} \sigma^{2}=L, \\
c_{11}^{2} u^{2}+c_{22}^{2} s^{2}=M, \\
c_{11} c_{44} u p+c_{22} c_{55} s \sigma \cos \psi=N,
\end{array}\right.
$$

oder

$$
\left\{\begin{aligned}
s & =\sqrt{f-f^{\prime} u^{2}}, \\
\sigma & =\sqrt{g-g^{\prime} u^{2}}, \\
s \sigma \cos \psi & =h-h^{\prime} u,
\end{aligned}\right.
$$

wenn $f, g, h, f^{\prime}, g^{\prime}, h^{\prime}$ Constanten bedeuten, die in gewisser Weise vol $L, M, N, p$ und den Grössen $c$ abhängen. Hieraus folgt:

$$
s \sigma \sin \psi=\sqrt{\left(f-f^{\prime} u^{2}\right)\left(g-g^{\prime} u^{2}\right)-\left(h-h^{\prime} u\right)^{2}}
$$

die Gleichungen (16.) und (17.) geben daher:

$$
\text { (20.) } \quad c_{22} d t=-c_{11} \frac{d u}{\sqrt{\left(f-f^{\prime} u^{2}\right)\left(g-g^{\prime} u^{2}\right)-\left(h-h^{\prime} u\right)^{2}}}
$$

und

$$
c_{22}^{2} d \varphi=c_{11} \frac{c_{11} u\left(h-h^{\prime} u\right)-c_{22} p\left(f-f^{\prime} u^{2}\right)}{\left(f-f^{\prime} u^{2}\right) \sqrt{\left(f-f^{\prime} u^{2}\right)\left(g-g^{\prime} u^{2}\right)-\left(h-h^{\prime} u\right)^{2}}} d u .
$$

Diese beiden Gleichungen erlauben $t$ und $\varphi$ durch elliptische Integrale als Functionen von $u$ darzustellen und $u$ und $\varphi$ mit Hulfe elliptischer Functionen durch $t$ auszudrücken. Ist das geschehen, so kann man durch Benutzung von (19.) und (15.) alle in (14.) vorkommenden Unbekaunten als Functionen von $t$ darstellen.

$$
\text { S: } 7
$$

Hat man bei einer beliebigen Gestalt des in der Flüssigkeit bewegten Körpers $u, v, w, p, q, r$ durch die Gleichungen (7.) als Functionen von $t$ be- 
254 Kirchhoff, über die Bevoegung eines Rotationskörpers in einer Flüssigkeit.

stimmt, so erfordert die Berechnung der eigentlichen Unbekannten des Problems, nämlich der Coordinaten $\alpha, \beta, \gamma$ und der Cosinus $\alpha_{1}, \beta_{1}, \gamma_{1}, \alpha_{2}, \beta_{2}, \gamma_{2}$, $\alpha_{3}, \beta_{3}, \gamma_{3}$ immer nur die Ausführung von Quadraturen.

Von den willkürlichen Constanten $A, B, C$, die in den Gleichungen (9.) vorkommen, kann man zwei, etwa $B$ und $C$, gleich Null setzen, ohne die Allgemeinheit der betrachteten Bewegung zu beeinträchtigen; man verfügt dadurch nur über die Richtung der $\xi$-Axe. Betrachtet man nämlich $\frac{\partial T}{\partial u}$, $\frac{\partial T}{\partial v}, \frac{\partial T}{\partial w}$ als die Componenten einer Geschwindigkeit nach den Axen der $x, y, z$, so zeigen die Gleichungen (9.), dass die Componenten dieser Geschwindigkeit nach den Axen der $\xi, \eta, \zeta$ den Constanten $A, B, C$ gleich sind; giebt man der $\xi$-Axe die Richtung dieser Geschwindigkeit, so wird $B=0$ und $C=0$. Zugleich wird nach (11.):

$$
A^{2}=M \text {. }
$$

Multiplicirt man nach dieser Festsetzung die Gleichungen (9.)

mit $\alpha_{1}$ oder mit $\alpha_{2}$ oder mit $\alpha_{3}$

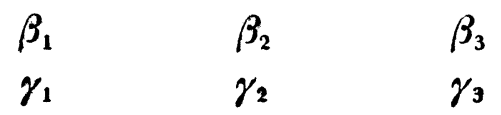

und addirt sie jedesmal, so erhält man:

$$
\alpha_{1}=\frac{1}{A} \frac{\partial T}{\partial u}, \quad \alpha_{2}=\frac{1}{A} \frac{\partial T}{\partial v}, \quad \alpha_{3}=\frac{1}{A} \frac{\partial T}{\partial v} .
$$

Um für die übrigen Cosinus Ausdrücke zu finden, setze man:

$$
\begin{aligned}
& \alpha_{1}=\cos \theta, \\
& \alpha_{2}=-\sin \Phi \sin \theta, \\
& \alpha_{3}=\cos \Phi \sin \theta, \\
& \beta_{1}=\sin \Psi \sin \theta, \\
& \beta_{2}=\cos \Phi \cos \Psi+\sin \Phi \sin \Psi \cos \theta, \\
& \beta_{3}=\sin \Phi \cos \Psi-\cos \Phi \sin \Psi \cos \theta, \\
& \gamma_{1}=-\cos \Psi \sin \theta, \\
& \gamma_{2}=\cos \Phi \sin \Psi-\sin \Phi \cos \Psi \cos \theta, \\
& \gamma_{3}=\sin \Phi \sin \Psi+\cos \Phi \cos \Psi \cos \theta
\end{aligned}
$$

Die Winkel $\theta$ und $\Phi$ sind dann aus den Werthen von $\alpha_{1}, \alpha_{2}, \alpha_{3}$ zu bestimmen, und es bleibt nur $\Psi$ zu ermitieln. Man hat aber

$$
\operatorname{tg} \Psi=-\frac{p_{1}}{n_{1}}
$$


Kirchhoff, ibber die Bewegung eines Rotationskörpers in' einer Flitssigkeit. 255 und, nach den Definitionsgleichungen von $p, q, r$ :

$$
\frac{d \beta_{1}}{d t}=\beta_{3} q-\beta_{2} r, \quad \frac{d \gamma_{1}}{d t}=\gamma_{3} q-\gamma_{2} r
$$

mit Hülfe hiervon findet man:

oder

$$
\frac{d \Psi}{d t}=-\frac{\alpha_{2} q+\alpha_{3} r}{\alpha_{2}^{2}+\alpha_{3}^{2}}
$$

$$
\text { (21.) } \frac{d \Psi}{d t}=-A \frac{q \frac{\partial T}{\partial v}+r \frac{\partial T}{\partial w}}{\left(\frac{\partial T}{\partial v}\right)^{2}+\left(\frac{\partial T}{\partial w}\right)^{2}} \text {. }
$$

Zur Bestimmung der Coordinaten $\beta$ und $\gamma$ führen die Gleichungen (10.). Die beiden letzten von diesen geben, wenn man die in ibnen vorkommenden Constanten $B$ und $I$ gleich Null setzt, was darauf hinauskammt, dass man über die Lage der $\boldsymbol{\xi}$-Axe verfügt:

$$
\begin{aligned}
& \gamma=\frac{1}{A}\left(\beta_{1} \frac{\partial T}{\partial p}+\beta_{2} \frac{\partial T}{\partial q}+\beta_{3} \frac{\partial T}{\partial r}\right), \\
& \beta=-\frac{1}{A}\left(\gamma_{1} \frac{\partial T}{\partial p}+\gamma_{2} \frac{\partial T}{\partial q}+\gamma_{3} \frac{\partial T}{\partial r}\right) .
\end{aligned}
$$

Die erste der Gleichungen (10.) führt auf die letzte der Gleichungen (8.) zurück. Die Ordinate $\alpha$ endlich ergiebt sich aus der Gleichung

$$
\frac{d \alpha}{d t}=\alpha_{1} u+\alpha_{2} v+\alpha_{3} v
$$

oder

$$
\text { (22.) } \frac{d \alpha}{d t}=\frac{1}{A}\left(u \frac{\partial T}{\partial u}+v \frac{\partial T}{\partial v}+v \frac{\partial T}{\partial v}\right) \text {. }
$$

In dem im vorigen Paragraphen behandelten Falle, dass der Korper ein Rotationskörper ist, kann man setzen:

$$
\begin{gathered}
\cos \theta=\frac{1}{A} c_{11} u, \quad \sin \theta=-\frac{1}{A} c_{22} \& \\
\Phi=\frac{\pi}{2}+\varphi
\end{gathered}
$$

die Gleichung (21.) wird:

die Gleichung (22.):

$$
\frac{d \Psi}{d t}=-\frac{\Lambda}{c_{2 \varepsilon}} \frac{h-h^{\prime} u}{f-f^{\prime} u^{2}},
$$

$$
\frac{d \alpha}{d t}=\frac{1}{A}\left(c_{22} f+\left(c_{11}-c_{21} f^{\prime}\right) u^{2}\right)
$$


256 Kirchhoff, über die Bewoegung eines Rotationskörpers in einer Fliissigkeit.

oder, da nach (18.) und (19.):

$$
\begin{gathered}
f=\frac{A^{2}}{c_{22}^{2}}, \quad f^{\prime}=\frac{c_{11}^{2}}{c_{22}^{2}}, \\
\text { (23.) } \frac{d \alpha}{d t}=\frac{A}{c_{22}}\left(1-c_{11}\left(c_{11}-c_{22}\right) \frac{u^{2}}{A^{2}}\right) .
\end{gathered}
$$

Führt man nun für $d t$ den aus (20.) sich ergebenden Werth ein, so erhält man auch $\Psi$ und $\alpha$ als elliptische Integrale durch $u$ ausgedrückt.

\section{S. 8.}

Lässt man die Constanten $h$ und $h^{\prime}$, die durch die Gleichungen (19.) eingeführt sind, oder, was auf dasselbe hinauskommt, die Constanten $p$ und $N$ verschwinden, so gelangt man zu dem, im Eingange angeführten, von den Herren Thomson und Tait behandelten Falle. Es wird dann $\varphi$ einer willkürlichen Constanten gleich; setzt man diese gleich Null, d. h. verfügt man bei gegebener Anfangsbewegung in gewisser Weise über die Richtung der $y$-Axe, so wird

$$
w=0, \quad q=0, \quad \Phi=\frac{\pi}{2} .
$$

Ferner wird auch $\Psi$ gleich einer willkürlichen Constanten; macht man diese gleich $\frac{\pi}{2}$, indem man die Richtung der $\eta$-Axe passend wählt, so hat man:

$$
\begin{array}{lll}
\alpha_{1}=\cos \theta, & \beta_{1}=\sin \theta, & \gamma_{1}=0, \\
\alpha_{2}=-\sin \theta, & \beta_{2}=\cos \theta, & \gamma_{2}=0, \\
\alpha_{3}=0, & \beta_{3}=0, & \gamma_{3}=1, \\
\cos \theta=\frac{1}{A} c_{11} u, & \sin \theta=-\frac{1}{A} c_{22} v
\end{array}
$$

Endlich findet man

$$
\gamma=0, \quad \beta=-\frac{1}{A} c_{55} r,
$$

während $\alpha$ aus Gleichụng (23.) zu berechnen ist.

Es hat keine Schwierigkeit für diesen Fall die in den beiden vorigen Paragraphen abgeleiteten Gleichungen weiter zu discutiren und durch sie die Unbekannten des Problems als elliptische Functionen der Zeit auszudrücken. Einfacher aber gelangt man zu demselben Ziele durch Betrachtungen, die sich an die Differentialgleichungen knüpfen, die in diesem Falle gelten. Setzt man in den Gleichungen (14.)

$$
w=0, \quad q=0, \quad q=0,
$$


so werden sie:

$$
\begin{aligned}
& c_{11} \frac{d u}{d t}=-c_{22} v r, \\
& c_{22} \frac{d v}{d t}=c_{11} u r, \\
& c_{55} \frac{d r}{d t}=-\left(c_{11}-c_{22}\right) u v .
\end{aligned}
$$

Vergleicht man dieselben mit den identischen Gleichungen:

$$
\begin{aligned}
& \frac{d \sin \operatorname{am} \lambda t}{d t}=\lambda \cos \operatorname{am} \lambda t \Delta \operatorname{am} \lambda t, \\
& \frac{d \cos \operatorname{an} \lambda t}{d t}=-\lambda \sin \operatorname{am} \lambda t \Delta \operatorname{am} \lambda t, \\
& \frac{d \Delta \operatorname{am} \lambda t}{d t}=-\lambda x^{2} \sin \operatorname{am} \lambda t \cos \operatorname{am} \lambda t,
\end{aligned}
$$

in denen $*$ den Modul der elliptischen Functionen bedeutet, so sieht man, dass bei passender Wahl des Anfangspunktes der Zeit die Grössen $u, v, r$ die Werthe $l \sin a m \lambda t, \quad m \cos \mathrm{am} \lambda t, \quad n \Delta \mathrm{am} \lambda t$

annehmen müssen, wenn die Constanten passend bestimmt werden. Diese Werthe können unter jene Grössen so vertheilt werden, dass alle Constanten reell sind und $\varkappa$ ein echter Bruch ist. Um das zu bewirken, gehe man von den Gleichungen

$$
\begin{array}{ll}
c_{11} u^{2}+c_{22} v^{2}+c_{55} r^{2} & =L, \\
c_{11}^{2} u^{2}+c_{22}^{2} v^{2} & =M
\end{array}
$$

aus, in welche sich durch die jetzt eingeführten Annahmen die Gleichungen (18.) verwandeln, und welche Integrale der jetzt vorliegenden Differentialgleichungen sind. Bedenkt man, dass, wenn die genannte Absicht erreicht ist, $\cos ^{2}$ am und $A^{2}$ am abnehmen, wenn $\sin ^{2}$ am wächst, so folgt aus der zweiten von diesen Gleichungen, dass eine von den beiden Grössen $u$ und $v$ durch sin am ausgedrückt werden muss, weil ihr zufolge $u^{2}$ und $v^{2}$ in entgegengesetztem Sinne sich gleichzeitig ändern. Aus den beiden Gleichungen ergiebt sich

$$
\left\{\begin{array}{l}
c_{11}\left(c_{22}-c_{11}\right) u^{2}+c_{22} c_{55} r^{2}=\text { Const. } \\
\text { und } \\
c_{22}\left(c_{11}-c_{22}\right) v^{2}+c_{11} c_{55} r^{2}=\text { Const. }
\end{array}\right.
$$

Da nun $c_{11}, c_{22}, c_{55}$ positive Grössen sind, so folgt aus derselben Eigenschaft der in Rede stehenden elliptischen Functionen, dass $u$ durch sin am ausgedrũckt werden muss, wenn $c_{22}>c_{11}$, und $v$, wenn $c_{11}>c_{22}$ ist. Jeder dieser beiden Fälle theilt sich wieder in zwei.

Journal für Mathematik Bd. LXXI. Heft 3. 
258 Kirchhoff, ibber die Bewegung eines Rotationskörpers in einer Flüsigkeit.

Ist $u$ durch sin am ausgedrückt, also $c_{22}>c_{11}$, so kann $v$ durch cosam und $r$ durch $\mathcal{A}$ am auszudrücken sein oder umgekehrt. Maassgebend ist dabei, dass $\cos ^{2}$ am für gewisse Werthe des reellen Arguments verschwindet, $A^{2}$ am aber nicht. Bezeichnen $v_{1}$ und $r_{1}$ die Werthe, die $v$ und $r$ zu einer beliebig gewählten Zeit besitzen, so ist nach (24.)

$$
-c_{22}\left(c_{22}-c_{11}\right) v^{2}+c_{11} c_{55} r^{2}=-c_{22}\left(c_{22}-c_{11}\right) v_{1}^{2}+c_{11} c_{55} r_{1}^{2} \text {. }
$$

Es kann hiernach $v^{2}$ verschwinden und $r^{2}$ kann nicht verschwinden, sobald der Ausdruck auf der rechten Seite dieser Gleichung pasitiv ist; das Umgekehrte findet statt, sobald dieser Ausdruck negativ ist; im ersten Falle ist $v$, im zweiten $r$ durch cos am auszudrücken.

Eine ähnliche Betrachtung ist auf den Fall, dass $c_{11}>c_{22}$ ist, anwendbar.

Es sollen für die vier Fälle, die hiernach zu unterscheiden sind, die Formeln angegeben werden, welche die Unbekannten des Problems in reeller Form als Functionen der Zeit darstellen. Dabei sollen durch $u_{0}, v_{0}, r_{0}$ die Werthe von $u, v, w$ für $t=0$ bezeichnet werden. Eine von den Grössen $u_{10}, v_{0}$ ist dann Null, die andere und $r_{0}$ sind den Grōssen $m, n$ gleich, da, wenn das Argument verschwindet, $\sin a m=0$ und $\cos a m=\Delta a m=1$ ist.

\section{F a ll 1 .}

Wenn $c_{22}>c_{11}$ und $c_{11} c_{55} r_{1}^{2}>c_{22}\left(c_{22}-c_{11}\right) v_{1}^{2}$, so ist

$$
\begin{aligned}
& u=l \sin \operatorname{am} \lambda t, \quad c_{11} l=-\frac{c_{22} v_{0} r_{0}}{\lambda}, \\
& v=v_{0} \cos \operatorname{am} \lambda t, \quad c_{22} v_{1}=-\frac{c_{11} l r_{0}}{\lambda}, \\
& r=r_{0} \Delta \operatorname{am} \lambda t, \quad c_{i 5} r_{0}=-\frac{\left(c_{22}-c_{11}\right) l v_{0}}{\lambda x^{2}}, \\
& \varkappa^{2}=\frac{c_{22}\left(c_{22}-c_{11}\right) v_{0}^{2}}{c_{11} c_{55} r_{0}^{2}} \\
& \lambda=r_{0} \text {, } \\
& l=-\frac{c_{22}}{c_{11}} v_{11} \\
& A=c_{22} v_{0} \text {, } \\
& \theta=-\frac{\pi}{2}-\operatorname{am} \lambda t \text {, } \\
& \beta=-\frac{c_{35} r_{0}}{c_{22} v_{0}} \Delta \mathrm{am} \lambda t, \\
& \alpha=\frac{v_{0}}{r_{0}}\left\{\left(1+\frac{c_{55} r_{0}^{2}}{c_{22} v_{0}^{2}}\right) \lambda t-\frac{c_{55} r_{0}^{2}}{c_{22} v_{0}^{2}} E(\lambda t)\right\} \text {, }
\end{aligned}
$$


Kirchhoff, über die Bewegung eines Rotationskörpers in einer Flissigkeit. 259

wo

$$
E(u)=\int_{11}^{\cdot u} \mathscr{A}^{2} \text { am } u d u
$$

und $\alpha=0$ für $t=0$ angenommen ist.

\section{F a ll 2 .}

Wenn $c_{2 !}>c_{11}$ und $c_{22}\left(c_{22}-c_{11}\right) v_{1}^{2}>c_{11} c_{55} r_{1}^{2}$, so ist:

$$
\begin{array}{rc}
u=l \sin \operatorname{am} \lambda t, & c_{11} l=-\frac{c_{22} v_{0} r_{0}}{\lambda}, \\
v=v_{0} d \operatorname{am} \lambda t, & c_{22} v_{0}=-\frac{c_{11} l r_{0}}{\lambda x^{2}}, \\
r=r_{10} \cos \operatorname{am} \lambda t, & c_{55} r_{1}=-\frac{\left(c_{22}-c_{11}\right) l v_{0}}{\lambda}, \\
\varkappa^{2} & =\frac{c_{11} c_{55} r_{0}^{2}}{c_{22}\left(c_{22}-c_{11}\right) v_{0}^{2}}, \\
\lambda & =\frac{r_{0}}{x}, \\
l & =-\varkappa \frac{c_{22}}{c_{11}} v_{10}, \\
A & =c_{22} v_{10},
\end{array}
$$$$
c_{22} v_{0}=-\frac{c_{11} l r_{0}}{\lambda x^{2}}
$$

$\cos \theta=-\varkappa \sin \operatorname{am} \lambda t, \quad \cdot \sin \theta=-\Delta \operatorname{am} \lambda t$,

$$
\begin{gathered}
\beta=-\frac{c_{55} r_{11}}{c_{22} v_{0}} \cos \mathrm{am} \lambda t, \\
\alpha=\sqrt{\frac{c_{22} c_{55}}{c_{11}\left(c_{22}-c_{11}\right)}}\left(\lambda t-\frac{c_{22}-c_{11}}{c_{22}} E(\lambda t)\right) .
\end{gathered}
$$

\section{F a 113.}

Wenn $c_{11}>c_{22}$ und $c_{22} c_{55} r_{1}^{2}>c_{11}\left(c_{11}-c_{22}\right) u_{1}^{2}$, so ist

$$
\begin{aligned}
& u=u_{0} \cos \operatorname{am} \lambda t, \\
& c_{11} u_{0}=\frac{c_{22} l r_{0}}{\lambda}, \\
& 0=l \sin \mathrm{am} \lambda t, \\
& c_{22} l=\frac{c_{11} u_{0} r_{0}}{\lambda}, \\
& r=r_{0} \Delta \operatorname{am} \lambda t, \\
& c_{55} r_{0}=\frac{\left(c_{11}-c_{22}\right) u_{0} l}{\lambda *^{2} .}, \\
& x^{2}=\frac{c_{11}\left(c_{11}-c_{22}\right) u_{0}^{2}}{c_{28} c_{b 5} r_{0}^{2}}, \\
& \lambda=r_{\psi_{2}}
\end{aligned}
$$


260 Kirchhoff, über die Bewegung eines Rotationskörpers in einer Flïssigkeit.

$$
\begin{gathered}
l=\frac{c_{11}}{c_{22}} u_{0}, \\
A=c_{11} u_{0}, \\
\theta=-\mathrm{am} \lambda t, \\
\beta=-\frac{c_{55} r_{0}}{c_{11} u_{0}} \Delta \operatorname{am} \lambda t, \\
\alpha=\frac{u_{0}}{r_{0}}\left\{\left(1+\frac{c_{55} r_{0}^{2}}{c_{11} u_{0}^{2}}\right) \lambda t-\frac{c_{55} r_{0}^{2}}{c_{11} u_{0}^{2}} E(\lambda t)\right\} .
\end{gathered}
$$

\section{F a 114.}

Wenn $c_{11}>c_{22}$ und $c_{11}\left(c_{11}-c_{22}\right) u_{1}^{2}>c_{22} c_{55} r_{1}^{2}$, so ist

$$
\begin{gathered}
u=u_{0} \Delta \operatorname{am} \lambda t, \quad c_{11} u_{0}=\frac{c_{22} l r_{0}}{\lambda x^{2}}, \\
v=l \sin \mathrm{am} \lambda t, \quad c_{22} l=\frac{c_{11} u_{0} r_{0}}{\lambda}, \\
r=r_{10} \cos \mathrm{am} \lambda t, \quad c_{55} r_{0}=\frac{\left(c_{11}-c_{22}\right) u_{0} l}{\lambda}, \\
\varkappa^{2}=\frac{c_{22} c_{55} r_{0}^{2}}{c_{11}\left(c_{11}-c_{22}\right) u_{0}^{2}}, \\
\lambda=\frac{r_{0}}{x}, \\
l=\varkappa \frac{c_{11} u_{0}}{c_{22}}, \\
\cos \theta=\frac{c_{11} u_{0},}{\Delta \mathrm{am} \lambda t,} \sin \theta=-\varkappa \sin \mathrm{am} \lambda t, \\
\beta=-\frac{c_{55} r_{0}}{c_{11} u_{0}} \cos \mathrm{am} \lambda t, \\
\alpha=\sqrt{\frac{c_{11} c_{55}}{c_{22}\left(c_{11}-c_{22}\right)}\left\{\lambda t-\frac{c_{11}-c_{22}}{c_{11}} E(\lambda t)\right\} .}
\end{gathered}
$$

§. 9.

Noch ein zweiter specieller Fall des hier behandelten Problems möge erwähnt werden.

Die Differentialgleichungen, die aus (14.) durch die Substitution (15.) entstehen, und die oben nur theilweise angegeben sind, sind vollständig diese: 


$$
\begin{aligned}
c_{11} \frac{d u}{d t} & =-c_{22} s \sigma \sin \psi, \\
c_{22} \frac{d s}{d t} & =c_{11} u \sigma \sin \psi, \\
c_{55} \frac{d \sigma}{d t} & =-\left(c_{11}-c_{22}\right) u s \sin \psi \\
c_{22} \frac{d \varphi}{d t} & =-c_{11} u \frac{\sigma}{s} \cos \psi+c_{22} p \\
c_{22} c_{55} \frac{\partial \psi}{\partial t} & =u \cos \psi\left(c_{11} c_{55} \frac{\sigma}{s}-c_{22}\left(c_{11}-c_{22}\right) \frac{s}{\sigma}\right)-c_{22} c_{44} p .
\end{aligned}
$$

Eine particuläre Lösung derselben erhält man, wenn man $\psi$ gleich Null, ferner $u, s, \sigma$ gleich Constanten, die der Gleichung

$$
u\left(c_{11} c_{55} \frac{\sigma}{s}-c_{22}\left(c_{11}-c_{22}\right) \frac{s}{\sigma}\right)=c_{22} c_{44} p
$$

genügen, und

$$
\varphi=\left(p-\frac{c_{11} u \sigma}{c_{22} s}\right) t
$$

setzt. Man hat dann weiter:

$$
\begin{gathered}
A=\sqrt{c_{11}^{2} u^{2}+c_{22}^{2} s^{2}} \\
\cos \theta=\frac{c_{11} u}{A}, \quad \sin \theta=-\frac{c_{22} s}{A}, \\
\Phi=\frac{\pi}{2}+\varphi \\
\Psi=-\frac{A \sigma}{c_{22} s} t \\
\alpha=\frac{1}{A}\left(c_{11} u^{2}+c_{22} s^{2}\right) t, \\
\beta=\frac{1}{A^{2}}\left(c_{11} c_{55} u \sigma-c_{22} c_{44} s p\right) \cos \Psi \\
\gamma=\frac{1}{A^{2}}\left(c_{11} c_{55} u \sigma-c_{22} c_{44} s p\right) \sin \Psi .
\end{gathered}
$$

Aus diesen Ausdrücken von $\alpha, \beta, \gamma$ folgt, dass der Anfangspunkt der $x, y, z$ mit gleichbleibender Geschwindigkeit eine Schraubenlinie durchläuft, deren Axe die $\boldsymbol{\xi}$-Axe ist. Nennt man $\boldsymbol{R}$ den Radius der Cylinderfläche, auf der die Schraubenlinie liegt, so ist:

$$
R= \pm \frac{1}{A^{2}}\left(c_{11} c_{55} u \sigma-c_{22} c_{44} s p\right)
$$


oder wegen der Relation, die zwischen $u, s, p, \sigma$ bestehen soll:

$$
R= \pm \frac{1}{A^{2}} c_{22}\left(c_{11}-c_{: 2}\right) \frac{u s^{2}}{\sigma} .
$$

Die Geschwindigkeitscomponente des genannten Punktes senkrecht zur $\xi$-Axe ist daher

$$
\pm \frac{1}{A}\left(c_{11}-c_{22}\right) u s
$$

Bezeichnet man durch $\mu$ die Tangente des Winkels, den die Richtung eines Elementes der Schraubenlinie mit der Richtung der $\xi$-Axe bildet, so ist hiernach

$$
\pm \mu=\frac{\left(c_{11}-c_{22}\right) u s}{c_{11} u^{2}+c_{22} s^{2}} \text {. }
$$

Soll $u$ einen gegebenen Werth haben, so ist das Verhältniss $u: s$ aus dieser Gleichung zu bestimmen; dieselbe hat zwei reelle Wurzeln, sobald

$$
\mu^{2}<\frac{\left(c_{11}-c_{22}\right)^{2}}{4 c_{11} c_{22}} .
$$

Ist weiter der Radius $R$ gegeben, so dient die für diesen aufgestellte Gleichung zur Bestimmung von $\sigma: s$. Die zwischen $u, s, p, \sigma$ angenommene Relation lehrt dann $p: u$ kennen, so dass die Verhältnisse der genannten vier Grössen bestimmt sind. Diese können selbst berechnet werden, sobald noch die Geschwindigkeit, mit der die Schraubenlinie durchlaufen wird, gegeben ist. Man sieht, dass die Realität ihrer Werthe allein durch die Ungleichheit bedingt ist, die erfüllt sein muss, damit das Verhältniss $s: u$ reell ist.

Die in diesem Paragraphen angegebenen Resultate sind aus den Differentialgleichungen (14.) hergeleitet; sie lassen sich auch herleiten aus den Integralgleichungen, die im $\$$. 6 entwickelt sind. Zu diesem Zwecke hat man die Relationen zwischen den Constanten der Integration aufzustellen, die erfüllt sein müssen, damit die Gleichung für $u$, die man erhält, wenn man den Ausdruck unter dem Wurzelzeìchen in Gleichung (20.) gleich Null setzt, zwei gleiche Wurzeln hat, die dem Anfangswerthe von $u$ gleich sind.

Heidelberg, im November 1869. 